\title{
Achieving Nanoscale EDS Analysis in Transmission Scanning Electron Microscopy
}

\author{
Markus Boese ${ }^{1}$, Luyang Han $^{2}$, Fang Zhou ${ }^{2}$, Fabián Pérez Willard ${ }^{2}$ and Benjamin Tordoff ${ }^{2}$ \\ ${ }^{1}$ Carl Zeiss Microscopy GmbH, Oberkochen, Wisconsin, United States, ${ }^{2}$ Carl Zeiss Microscopy GmbH, \\ Oberkochen, Baden-Wurttemberg, Germany
}

The spatial resolution of energy dispersive spectroscopy (EDS) analysis is fundamentally limited by the interaction volume of characteristic X-ray emission. By using a thin specimen, the interaction volume can be reduced to nanometer scale in transmission scanning electron microcopy (STEM) in SEM, achieving nanometer scale resolution for EDS analysis. In this paper the details and caveats of STEM-EDS analysis will be discussed. Typical EDS analysis in SEM is performed at relatively high energy ( $>10 \mathrm{kV}$ ), leading to huge interaction volumes in order of micrometers. By reducing the energy of the primary electron beam, the interaction volume can be significantly reduced. With the help of a specially designed detector, EDS analysis with beam energy down to $1 \mathrm{kV}$ is feasible, demonstrating nanometer scale resolution [1]. However, low energy EDS is faced with many practical challenges: X-ray emission with low electron beam energy has very low yield, leading to low count rates during analysis. Moreover, for higher atomic numbers, low energy X-ray lines such as $\mathrm{M}$ and even $\mathrm{N}$ lines must be used. These low energy X-ray emission lines typically have complicated shapes and are very difficult to quantify. The high surface sensitivity of low energy EDS also requires a very clean specimen. Another method to reduce the interaction volume is to use a thin sample: in transmission scanning electron microscopy, sample thickness is reduced to tens of nanometers to be transparent to the electron beam. In contrary to low energy EDS, STEM-EDS benefits from the high energy of the primary electron beam to excite K or L lines, which can be easily quantified. A practical challenge for STEM-EDS is the stray X-ray signal. In the STEM configuration, the majority of the electrons penetrate through the specimen and arrive at various parts of the SEM system. This would produce pronounced stray X-ray signals especially from Al and Si K lines, originated from the SEM stage and the STEM detectors. To reduce the stray X-ray signal, a collimator should be used for the EDS detector to limit the acceptance angle of the incoming X-rays. Additionally, the specimen holder for STEM can be utilized to block the stray X-ray signal. This method is illustrated in Figure 2. There are multiple possible configurations to place the STEM specimen against the STEM detector, but only one optimum configuration to block the stray X-ray signal. In this favored configuration, the STEM specimen holder sits right below the EDS detector and above the STEM detector, thus blocking the direct line-of-sight between the EDS detector and rest of the SEM chamber and prevents the stray Xrays from entering the EDS detector. The geometry of this favored configuration in a ZEISS GeminiSEM can be seen in Figure 1. The STEM-EDS technique is especially suitable for nanomaterials. Due to their nanometer size in at least one of their dimensions, they are naturally transparent to high energy electron beam, thus can be prepared by simply dispersing them on a typical copper grid with carbon film. In the example shown in Figure 2, the Co nanoparticles dispersed in mesoporous silica are imaged at $30 \mathrm{kV}$ in high angular dark field mode. The Co nanoparticles have an average size of around $10 \mathrm{~nm}$ and are deposited inside the nanometer sized channels of the mesoporous silica. The elemental mapping shown in the figure demonstrates the capability of STEM-EDS to resolve structures even below $10 \mathrm{~nm}$. 


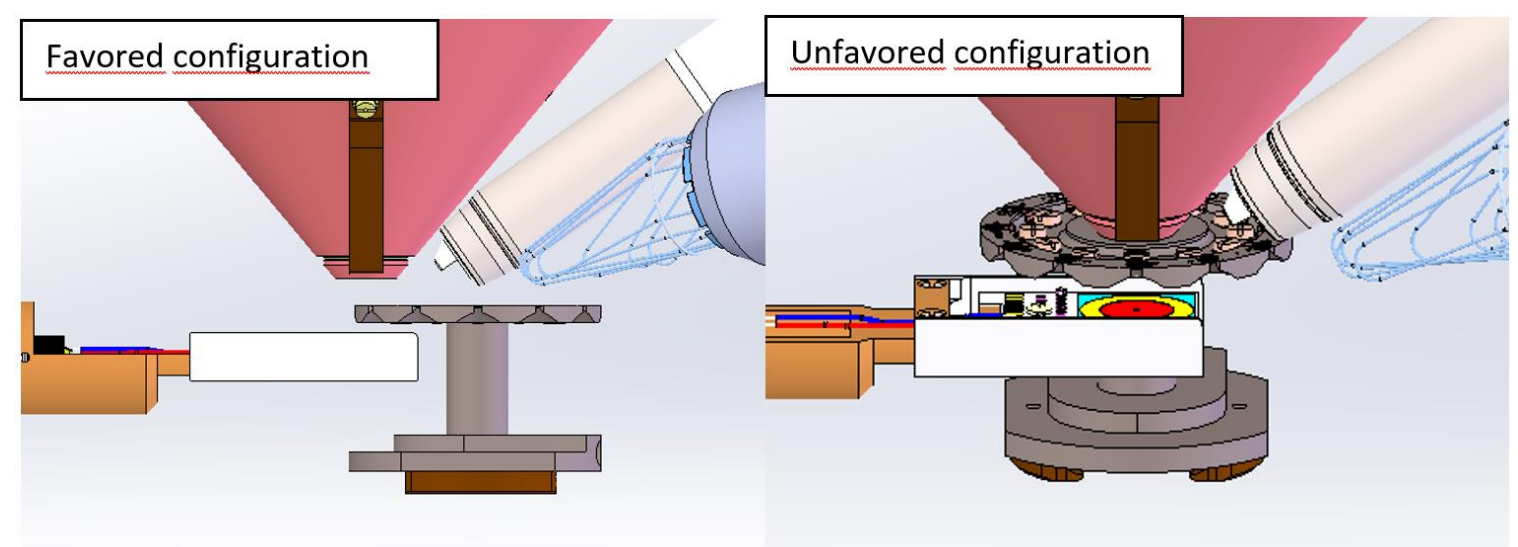

Figure 1. Comparing a favored (left) and unfavored (right) configuration for EDS analysis in STEM. Under the favored configuration, the stray X-ray signal from the detector and sample chamber is blocked by the STEM sample holder.

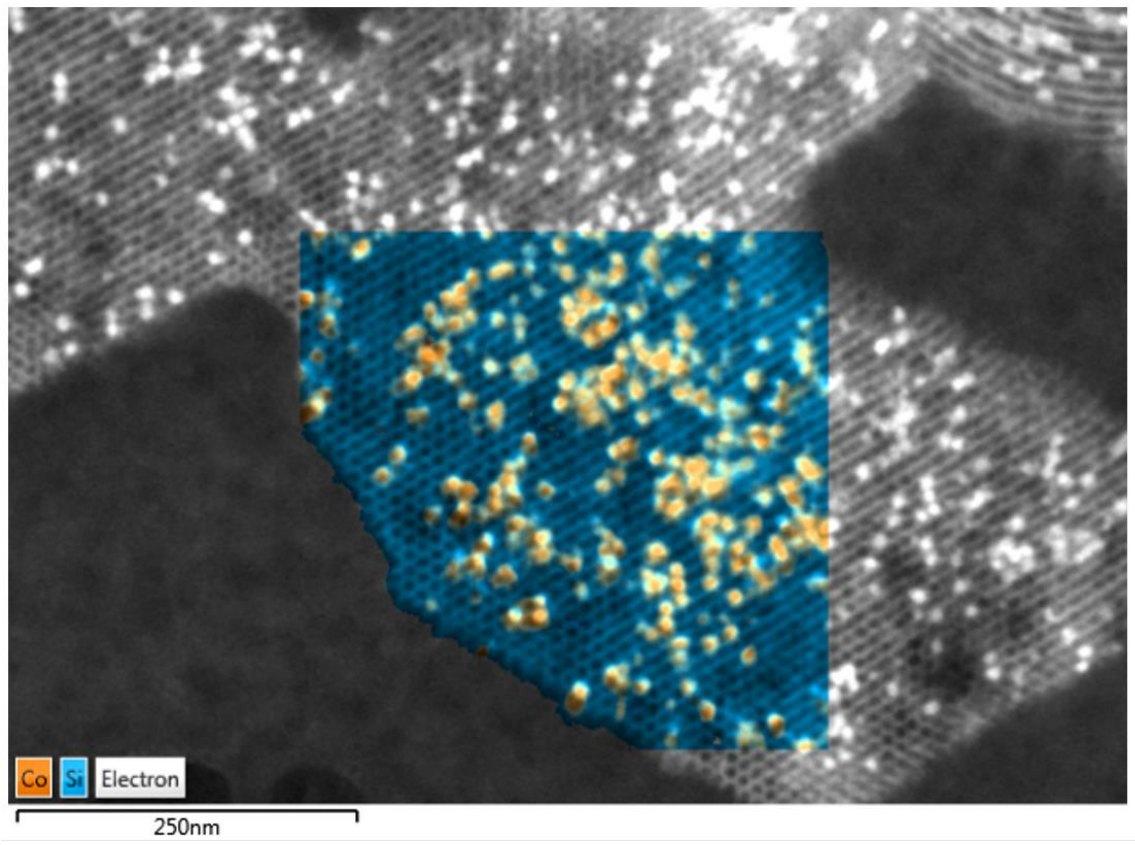

Figure 2. High resolution EDS mapping of Co nanoparticles embedded in mesoporous silica measured at $30 \mathrm{kV}$. Individual Co nanoparticles with approx. $10 \mathrm{~nm}$ size are resolved.

\section{References}

[1] Nanometer scale EDS Analysis using Low-kV FE-SEM and Windowless EDS Detector, Technology Note Zeiss. 